ESJ Social Sciences

\title{
Violences liées aux accusations de sorcellerie et vulnérabilité de la femme âgée en contexte burkinabé
}

\author{
Badolo Bawala Léopold \\ Université Joseph Ki-Zerbo,
} Département de Philosophie et de Psychologie, Ouagadougou, Burkina Faso

Doi:10.19044/esj.2021.v17n38p43

Submitted: 05 August 2021

Accepted: 12 November 2021

Published: 30 November 2021
Copyright 2021 Author(s)

Under Creative Commons BY-NC-ND

4.0 OPEN ACCESS

Cite As:

Léopold B.B. (2021). Violences liées aux accusations de sorcellerie et vulnérabilité de la femme âgée en contexte burkinabé. European Scientific Journal, ESJ, 17 (38), 43.

https://doi.org/10.19044/esj.2021.v17n38p43

\section{Résumé}

Cette recherche s'inscrit dans le cadre des études sur les violences psychologiques et sociales au Burkina Faso. L'objectif vise à appréhender le lien entre les violences et la vulnérabilité psychologique chez la femme âgée. Soixante-deux femmes exclues de leur communauté suite à des accusations de sorcellerie ont été interrogées par questionnaire et par entretien. Les résultats mettent en évidence la multiplicité des violences vécues, associées à une vulnérabilité psychologique. Ces violences, plus fréquemment, prennent la forme d'une non approbation d'un acte positif en faveur des victimes, d'un refus de dénoncer un acte susceptible de nuire aux victimes ou de leur assurer une quelconque protection, d'insultes ou d'autres moqueries. Ces violences sont des facteurs de vulnérabilité psychologique se traduisant, notamment, par un sentiment d'abandon et un regard peu valorisant porté sur soi.

Mots-clés : Sorcellerie, vulnérabilité, psychologie, femmes, Burkina Faso 


\title{
Violence Linked to Accusations of Witchcraft and the Vulnerability of Elderly Women in the Burkinabé Context
}

\section{Badolo Bawala Léopold}

Université Joseph Ki-Zerbo, Département de Philosophie et de Psychologie, Ouagadougou, Burkina Faso

\begin{abstract}
This research is a part of other studies on psychological and social violence in Burkina Faso. This paper focuses on understanding the link between violence and psychological vulnerability in the context of elderly women. Sixty-two women who had been excluded from their communities on charges of witchcraft were interviewed using a questionnaire. The results highlight the multiplicity of lived violence associated with psychological vulnerability. More frequently, such violence takes the form of non-approval of a positive act in favor of the victims, refusal to report an act likely to harm the victims or provide them with any protection, insults or other mockery. This violence is one of the factors of psychological vulnerability, resulting in a feeling of abandonment and a lack of self-esteem.
\end{abstract}

Keywords: Witchcraft, vulnerability, psychology, women, Burkina Faso

\section{Introduction}

La vulnérabilité traduit une faiblesse ou une déficience à partir de laquelle l'intégrité d'un être ou d'un lieu se trouve menacée d'être détruite, diminuée ou altérée. C'est un état de moindre résistance aux nuisances et aux agressions et de plus grande sensibilité à l'adversité (Brodiez-Dolino, 2013). Les personnes vulnérables sont celles qui sont menacées dans leur autonomie, leur dignité ou leur intégrité physique ou psychique. Il s'agit d'une « rupture d'équilibre d'un système dans une combinaison extrêmement complexe et labile de risques (de fragilités), de forces et de faiblesses, d'aléas physiques, psychologiques et environnementaux » (Bouisson, 2011, p.70). La vulnérabilité peut être temporaire ou chronique et varie d'un sujet à l'autre (variabilité interindividuelle). Chez un même sujet, elle varie au cours de la vie (variabilité intra-individuelle). Chez le sujet âgé, elle intègre des dimensions multiples endophénotypiques (génétiques, épigénétiques), diachroniques (traumas précoces, expériences de vie passées) et synchroniques (facteurs de stress actuels liés à la transition vers le grand âge) (Clery-Melin, 2014). Pour Fasse et al. (2012), la vulnérabilité chez les personnes plus âgées se manifeste sous forme de symptomatologie dépressive et de vécu de fardeau. Les sujets âgés constituent une population vulnérable, 
présentant plus de risque de développer des troubles psychopathologiques (Airagnes, 2014). La vulnérabilité peut résulter de l'âge, de la maladie, de l'infirmité, de la grossesse, d'une catastrophe naturelle ou de la violence liée à l'exclusion sociale.

Pour Mbanzoulou (2004), la violence est un abus de la force portant atteinte à un symbole, une chose ou une personne. Elle se décline en de multiples formes et selon différentes conditions (Sorel \& Dieu, 2013). Elle est inextricablement liée à l'Histoire de l'Humanité et constitue une composante essentielle de la nature humaine (Flynn et al., 2014). Une des formes que prend ce phénomène est ce que l'on appelle « la violence faite aux femmes ». Matlin (2007) note qu'elle inclut des actes intentionnels (physiques, psychologiques ou sexuels) qui visent à blesser. Selon la Déclaration sur l'élimination de la violence à l'égard des femmes adoptée par l'Assemblée générale des Nations Unies en décembre 1993, cette violence s'applique à «tous les actes de violences dirigés contre le sexe féminin, et causant ou pouvant causer aux femmes un préjudice ou des souffrances physiques, sexuelles ou psychologiques, y compris la menace de tels actes, la contrainte ou la privation arbitraire de liberté, que ce soit dans la vie publique ou dans la vie privée ».

Le présent article aborde les violences consécutives aux accusations de sorcellerie et la vulnérabilité qui en découle. On définit, dans un premier point, la problématique qui fonde la présente recherche. Un deuxième point du présent travail présente la méthode de recueil des données. Les résultats obtenus sont ensuite présentés et discutés.

\section{Problématique}

En dépit de l'évolution du droit et des progrès scientifiques, les femmes de tout âge continuent d'être victimes de violences (mutilations génitales féminines, violences conjugales, exclusion...). Un des phénomènes qui participe de ces violences est la sorcellerie et/ou la croyance en la sorcellerie. Erny (1979) en relève le caractère universel, avec cependant une variabilité des modalités et de l'intensité des menaces d'un pays ou d'une région à l'autre. Ainsi, le mode de fonctionnement de la sorcellerie, la façon de devenir sorcier et le processus d'ensorcellement, la façon de découvrir le sorcier, de le punir ou de le désintoxiquer varient d'un milieu culturel à un autre (Van Caeneghem, 1955). La croyance en la sorcellerie comme pratique de rites magiques afin de nuire, de porter atteinte aux personnes et aux biens, est une réalité sociologique perpétuée par la fidélité à des traditions surannées (Boa, 2010). L'intemporalité de cette croyance incite à une certaine prudence dans les jugements y relatifs. Pour Erny (1979), il ne faut pas prendre à la légère et balayer d'un revers de la main quelque chose en quoi des milliards d'hommes, aujourd'hui encore, attachent une importance primordiale. Pour lui, il y a dans le mot « sorcellerie » l'idée qu'une personne peut exercer une influence (sans 
intermédiaires visibles) sur d'autres. C'est une influence néfaste, maléfique, qui s'attaque à l'intégrité corporelle et mentale, à la famille, et à tout ce qui appartient à la personne visée. Par les procédés de jettature, la victime est mise aux prises avec la maladie, la malchance, l'oppression, le trouble, l'inefficacité et l'absence de vitalité qui la conduisent irréversiblement à la mort sociale voire physique. Les accusations de sorcellerie restent persistantes dans les pays du tiers-monde et surtout en Afrique. Tous les événements négatifs ou malheureux vécus par l'homme (maladies, accidents, morsures de bêtes, décès, manque de réussite, perte d'emploi, échecs aux examens ou concours, échec familial, mauvais comportement et délinquance d'enfant, etc.) sont attribuables à des forces occultes maléfiques déclenchées par les sorciers (Amselle, 2014 ; Boa, 2010). Dans le même sens, Fancello (2015) observe que les chaînes de violence sorcellaire découlant des accusations de sorcellerie constituent un aspect important et relativement récent de la crise sécuritaire que traversent certains pays africains. La rapidité des changements sociaux, les crises politiques, les guerres et conflits de tous ordres, ainsi que la vitalité religieuse dans les sociétés africaines, entraînent de nouvelles formes d'accusations, notamment à l'égard des populations les plus vulnérables (étrangers, femmes, personnes âgées, enfants, infirmes) provoquant à leur tour de nouvelles cascades de violences et invitant à sans cesse réactualiser le regard sur la sorcellerie (Fancello \& Bonhomme, 2018).

$\mathrm{Au}$ Burkina Faso, les croyances en la sorcellerie sont vivaces. En décembre 2016, neuf cent vingt-six (926) personnes, en provenance des contrées du centre du pays, pour la plupart des femmes exclues sociales suite à des accusations de sorcellerie portées contre elles, ont été enregistrées dans 13 centres d'accueil (encore appelés cours de solidarité) et seulement soixanteun (61) en parcours de retour en famille. En mi-mars 2018, le Centre Delwendé (mis en place par l'Eglise catholique) de Sakoula à lui seul comptait deux cent dix-sept (217) pensionnaires, accusés de sorcellerie, soit cinq (05) hommes et deux cent douze (212) femmes. Il s'agit de femmes d'âge avancé, vivant souvent seules et sans soutien social. Différents procédés sont utilisés pour détecter "un mangeur ou une mangeuse d'âmes" : recours aux devins, breuvage de "vérité", port du cadavre, aveu de culpabilité...

L'ampleur du phénomène d'accusation de sorcellerie et les violences exercées sur les personnes ont amené le gouvernement du Burkina Faso à adopter des instruments juridiques de protection des victimes. Par exemple, le pays a ratifié les instruments internationaux suivants :

- la Déclaration de 1967 sur l'élimination de la discrimination à l'égard des femmes ;

- la Déclaration sur l'élimination de la violence à l'égard des femmes de 1967 ; 
- le Protocole à la Charte Africaine des droits de l'homme et des peuples, relatif aux droits de la femme en Afrique, le 11 juillet 2003, à Maputo (Mozambique).

À côté de l'État, il y a d'autres acteurs (Médias, Associations, Organismes Non Gouvernementaux...) qui œuvrent dans la sensibilisation, l'information, la formation, en vue de lutter contre l'exclusion sociale et pour la réinsertion sociale des victimes.

D'un point de vue fondamental et théorique, des approches variées ont été développées pour décrire et comprendre le phénomène de violence. Martinelli et Bouju (2012) ont décrit les différentes violences que les accusations de sorcellerie déchaînent : violence de celui qui se croit promis à tous les échecs sociaux; violences populaires qui vont de la torture au lynchage; violences juridiques (longues incarcérations). Il y a aussi les violences religieuses, exigeant des aveux, jeûnes et purifications, et les violences familiales, pouvant entraîner jusqu'au bannissement et au meurtre (Roxburgh, 2016).

En psychologie, Fontaine (2003, cité par Sorel et Dieu, 2013) décrit la violence à partir de trois variables bimodales: le degré d'activité de l'agresseur (actif vs passif), le type de violence (physique vs verbale ou psychologique) et l'orientation de la violence (directe vs indirecte). La combinaison de ces variables et de leurs modalités permet de décliner la violence et l'agressivité en huit dimensions reprises dans le tableau ci-après.

Tableau 1. Taxonomie des comportements violents

\begin{tabular}{|l|l|l|l|}
\hline $\begin{array}{l}\text { Degré } \\
\text { d'activité }\end{array}$ & Type de violence & Orientation & Comportements \\
\hline \multirow{4}{*}{ Actif } & \multirow{2}{*}{ Physique } & Directe & Coups et blessures sur une victime \\
\cline { 2 - 4 } & Indirecte & $\begin{array}{l}\text { Coups et blessures sur un proche de } \\
\text { la victime }\end{array}$ \\
\cline { 2 - 4 } & $\begin{array}{l}\text { Verbale, } \\
\text { psychologique }\end{array}$ & Directe & Insultes, moqueries, harcèlement \\
\cline { 3 - 4 } & \multirow{3}{*}{ Passif } & Directe & $\begin{array}{l}\text { Empêcher la victime d'obtenir } \\
\text { quelque chose de positif }\end{array}$ \\
\cline { 2 - 4 } & $\begin{array}{l}\text { Verbale, } \\
\text { psychologique }\end{array}$ & Indirecte & Ne pas protéger la victime \\
\cline { 2 - 4 } & & Indirecte & $\begin{array}{l}\text { Ne pas dénoncer un acte nuisible à } \\
\text { une victime }\end{array}$ \\
\hline & & $\begin{array}{l}\text { Ne pas approuver un acte positif pour } \\
\text { une victime }\end{array}$ \\
\hline
\end{tabular}

Source : Fontaine (2003 cité par Sorel et Dieu, 2013, p. 156)

La violence psychologique consiste à exercer un contrôle psychologique sur la victime en l'humiliant ou en la dénigrant, en adoptant des propos et une attitude méprisante, en surveillant ses allées et venues ou les personnes qu'elle fréquente, en négligeant les besoins affectifs de la victime, 
en isolant cette dernière de son entourage ou en exerçant du chantage (RobertOuvray, 2008b). Ce type de violence peut nuire à l'estime de soi et à l'épanouissement social de la victime, compromettre sa capacité de percevoir, de sentir, de comprendre et d'exprimer des émotions (Hicks, 2017). Elle peut entraîner de graves problèmes sur le plan des émotions et du comportement : dépression, absence d'attaches affectives ou émotionnelles, faible capacité cognitive, résultats scolaires ou professionnels médiocres et compétences sociales sous-développées (Robert-Ouvray, 2008b).

La violence verbale est souvent associée à la violence psychologique et s'exerce à travers les mots. L'agresseur insulte et/ou crie lorsqu'il s'adresse à la victime. L'agression verbale (cris, insultes) ou symbolique (silence systématique) peut avoir des effets néfastes sur la victime.

La personne qui utilise la violence physique exerce la force physique (coups, gifles, bousculades, brûlures, étranglement ou emploi d'une arme) pour obtenir ce qu'elle veut (Robert-Ouvray, 2008a).

Sorel et Dieu (2013) proposent un modèle bio-psycho-social qui questionne l'acte de violence d'un point de vue à la fois bio-logique, psychologique et socio-logique. Ce modèle permet de situer la responsabilité plurielle dans tout acte de violence. Dans ce modèle, le premier responsable direct est l'auteur de la violence, qui n'a pas réussi à se défaire de sa propension naturelle à agresser ses pairs. "L'individu est responsable de ses gènes, des conduites à risque dans lesquelles il s'engage, de son manque d'inhibition ou de son répertoire comportemental pauvre » (Sorel \& Dieu, 2013, p.162). Du côté de la victime, il peut paraître a priori délicat et gênant de considérer qu'elle est responsable de la violence subie. En s'abstenant de procéder ainsi, on évite de la culpabiliser et de la rendre responsable. Cependant, « la victime n'a malheureusement pas réussi à dissuader l'auteur de s'en prendre à elle » (Sorel \& Dieu, 2013, p.162). Elle est alors responsable de son manque de compétences sociales et du fait d'avoir été au mauvais endroit au mauvais moment. La responsabilité de l'environnement semble plus subtile et plus pernicieuse. La société a le devoir d'éduquer ses citoyens et de les guider sur le droit chemin. La violence constatée, et dont les individus sont auteurs et victimes, consacre un échec de la société qui n'a pas pu empêcher l'auteur de devenir violent et qui n'a pas pu protéger la victime.

La réalité de la violence dont les femmes sont victimes peut s'apprécier dans une perspective psycho-développementaliste. Le développement psychologique est un processus dynamique et continu qui perdure tout au long de la vie et qui est organisé autour d'étapes majeures que sont l'enfance, l'adolescence, l'adultescence, la maturescence et la sénescence (Richard, 2004). L'âge adulte est la période la plus longue de la vie. Les énergies physiques, intellectuelles, affectives y atteignent leurs sommets avant la lente descente vers la vieillesse. Pour Richard (2004) et Piazza (2008), le 
vieillissement est un processus dynamique et irréversible auquel est soumis l'organisme vivant de sa naissance à sa mort. Ce processus, en lui-même, cause moins de dommages que les maladies qui l'accompagnent (cancers, hypertension, problèmes cardio-pulmonaires, démences vasculaires...).

Les accusations de sorcellerie concernent aussi bien les enfants (Fancello, 2015) que les personnes âgées. Lorsqu'elles interviennent à un moment où la personne est avancée en âge, elles alourdissent le poids du vieillissement. Ces accusations sont nombreuses, injustes, et parfois fatales, pour la victime qui se trouve dans une position qui la rend impuissante à réagir devant la pression des accusateurs (Coakley, 2015). Selon Kibuga et Dianga (2000), en Tanzanie, en raison de la rareté des terres cultivables, il est plus difficile de gagner assez d'argent pour satisfaire aux différents besoins de la vie. Les bras valides émigrent en ville, laissant les membres plus âgés de la communauté seuls à la campagne. Ce problème touche plus les femmes que les hommes, en partie parce qu'elles ont tendance à vivre plus longtemps que les hommes et sont donc laissées comme veuves ; mais aussi parce que les hommes veufs sont susceptibles de se remarier à de plus jeunes femmes qui prendront soin d'eux. L'option du remariage est rarement ouverte aux femmes âgées. Elles doivent se battre quotidiennement pour satisfaire leurs besoins basiques. Aussi, en raison d'une mauvaise alimentation et/ou d'une maladie, elles n'ont pas toujours la force de quitter leurs maisons et un air de mystère ou d'austérité peut se développer autour d'elles ; ce qui contribue fortement aux accusations de sorcellerie. Les mauvaises conditions de vie et le combustible fumant avec lequel elles cuisinent contribuent à provoquer des rides et des yeux rouges, signes caractéristiques incontestables de sorcellerie dans les représentations populaires tanzaniennes (Kibuga \& Dianga, 2000). Par ailleurs, la vétusté de leurs maisons fait d'elles des cibles faciles pour les cambriolages. Seules et sans défense, elles ne disposent d'aucun moyen pour repousser les accusations et les attaques. Crampton (2013) conforte ce constat en montrant qu'au Ghana, des vieilles femmes sont accusées de sorcellerie et sont marginalisées, abusées et souvent assassinées. Il est rejoint par Owusu (2020) qui rapporte que dans la province du Limpopo en Afrique du Sud, environ 389 exécutions de personnes accusées de sorcellerie ont été recensées entre 1985 et 1995. De 1996 à 2001, dans la même province, 600 meurtres ont été enregistrés pour les mêmes raisons.

Dans le cadre du présent travail, on propose de s'intéresser à la période du troisième âge. Dans cette contribution, on tente particulièrement de répondre à la question suivante : quel est le rapport entre les violences liées aux accusations de sorcellerie et la vulnérabilité chez les femmes qui en sont victimes au Burkina Faso ? Dans le contexte étudié, l'absence de travaux sur cette question ne permet pas de prendre conscience des enjeux et des conséquences psychologiques des violences liées aux accusations de 
sorcellerie, d'où l'objet et l'intérêt de la présente recherche. On formule l'hypothèse que les violences liées aux accusations de sorcellerie constituent un facteur de vulnérabilité des personnes qui en sont victimes.

\section{Méthode}

\subsection{Personnes enquêtées}

Soixante-deux (62) femmes en situation d'exclusion sociale consécutive à une accusation de sorcellerie ont participé à cette recherche. Elles ont un âge moyen de 67 ans. Il s'agit de femmes autrefois mariées. Elles sont toutes mères. Certaines sont grand-mères.

\subsection{Instruments}

On a utilisé un questionnaire pour interroger toutes les participantes sur leurs expériences de l'exclusion sociale (avec ses deux dimensions principales que sont la séparation sociale significative et l'hystérésis de cette séparation; Trainar, 2006), faisant suite à l'accusation de sorcellerie. Le questionnaire a été construit à partir du tableau proposé par Sorel et Dieu (2013, p. 156), et a permis d'explorer le type de violence subie à la suite de l'accusation de sorcellerie. Des données qualitatives ont été recueillies par un guide d'entretien pour compléter celles obtenues par questionnaire. L'entretien a permis d'étudier le vécu psychologique de la violence subie et la manière dont elle interfère sur la vulnérabilisation des participantes. L'entretien n'a concerné qu'une partie d'entre elles.

\subsection{Procédure}

Les participantes ont été rencontrées à des moments différents dans les centres d'accueil des personnes exclues pour accusation de sorcellerie. Après leur avoir expliqué le but de cette recherche, la participation volontaire de chacune a été requise. Le questionnaire a été hétéro-administré en raison de l'analphabétisme des enquêtées qui ne pouvaient donc pas le remplir ellesmêmes. La durée moyenne d'administration du questionnaire a été estimée à vingt (20) minutes contre quinze (15) pour l'entretien. Des relances ou recadrages sont faits dans le souci d'amener l'interviewée à rester dans le champ de la question abordée.

\section{Résultats}

Les informations quantitatives sont analysées avec les données qualitatives. Après le dépouillement des réponses au questionnaire, on a généré, sous Excel, un graphique qui ressort les fréquences d'exposition des femmes accusées de sorcellerie à chaque forme de violence considérée. Les commentaires qui en sont faits sont appuyés par des extraits des entretiens 
réalisés avec certaines d'entre elles. Cela a permis de relever le lien entre violences subies et vulnérabilité.

\subsection{Accusations de sorcellerie et violences subies}

Les accusations de sorcellerie dont les personnes enquêtées ont été victimes ont entraîné une diversité de violences. Le graphique ci-après donne un aperçu de la répartition des violences qu'elles ont subies.

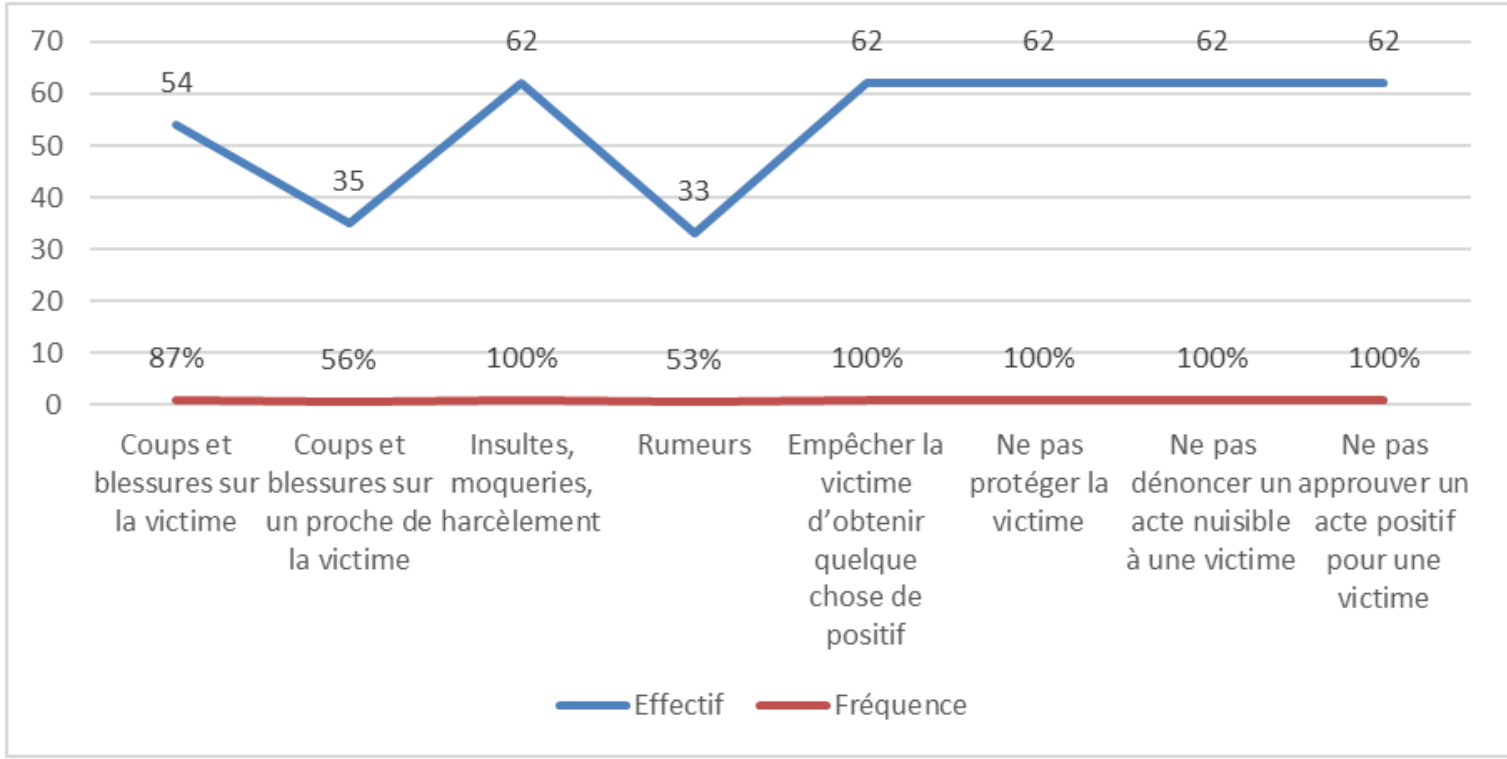

Graphique 1. Type de violence subie par les participantes

Ce graphique montre que les enquêtées ont été victimes de tous les types de violences (physique, verbale et psychologique) décrits par Sorel et Dieu (2013). Il montre aussi la présence des différentes orientations (directe vs indirecte) que prennent ces violences. Pour chaque type de violence, on observe une fréquence d'évocation élevée. Les formes de violences les plus répandues concernent :

- le fait de ne pas approuver un acte positif, quel qu'il soit, en faveur de la victime ;

- la non dénonciation d'un acte susceptible de nuire à la victime ;

- la non protection de la victime ;

- l'empêchement fait à la victime d'obtenir quelque chose de positif ;

- les insultes et autres moqueries.

Les formes relativement moins fréquentes concernent les rumeurs, les coups et blessures. Cette configuration des résultats appelle deux remarques :

- une fois l'accusation de sorcellerie manifestée, l'entourage retire toute assistance à la victime ;

- les accusations ont valeur de vérité. 
Les propos suivants illustrent la mécanique de l'accusation et de l'exclusion : «Une femme est décédée subitement dans notre cour. Tout de suite, les soupçons se sont dirigés vers moi parce que je ne m'entendais pas avec cette femme. Malgré mon innocence, on m'a accusée d'avoir tué cette femme. C'est très difficile à supporter. Les gens du quartier et ceux de la cour se sont réunis à trois reprises pour décider de mon bannissement. Même les enfants m'insultaient et se moquaient de moi. Le chef de la cour m'a donné jusqu'à midi pour disparaître du village, sinon je serai égorgée. Et je suis partie à la hâte, sans pouvoir prendre quoi que ce soit. » (H.Y. est une femme âgée d'environ soixante-six (66) ans, mère de cinq (05) enfants dont quatre garçons et une fille, vivant tous en Côte d'Ivoire). Ce témoignage décline la brutalité de l'accusation. C'est une violence en soi, accompagnée des autres formes de violence (insultes, moqueries, menaces de mort, bannissement). Les auteurs de ces violences sont les proches (les gens de la cour conjugale) et les gens du quartier. Cette femme n'a bénéficié d'aucune défense. Une autre victime témoigne : «Il y a un voisin qui est décédé dans le quartier. Nous avons dormi devant la porte du défunt. Le lendemain nous sommes allées au puits. De retour, $\mathrm{j}$ 'apprends que quelque chose s'est produit. Le rituel a désigné deux maisons : la mienne et celle de mon beau-frère. Trois jours après, on nous a demandé de partir. Mon mari a dit qu'on doit partir au risque d'être humiliés. Moi je suis venue à Ouagadougou et mon beau-frère est parti avec ses deux femmes » (K. P. S., âgée d'environ soixante (60) ans, mère de cinq (05) filles dont deux sont décédées après sa venue au centre). Ces propos illustrent le manque de fondement objectif pour soutenir les accusations de sorcellerie portées contre cette femme.

\subsection{Violences subies et vulnérabilité}

Les différents types de violences auxquelles les enquêtées ont été exposées à la suite des accusations de sorcellerie portées contre elles entretiennent un rapport avec la vulnérabilité.

Cette vulnérabilité se traduit, entre autres, par :

- une vie solitaire, avec peu de contacts avec la famille ou les proches ;

- une dépendance des agents du centre d'accueil pour les soins et la subsistance ;

- un état de confusion et d'hésitation observé chez certaines d'entre elles ;

- un sentiment de désespoir qui transparaît sur les visages...

Lorsque l'on se réfère au cas de H.Y., on observe qu'elle est déjà fragilisée par l'âge (66 ans) et par le fait que ses enfants vivent loin d'elle. Les accusations de sorcellerie portées contre elle et les désagréments qui ont suivi (insultes, moqueries, menaces de mort, bannissement) viennent alourdir le 
poids de cette fragilité. La brutalité des accusations et du bannissement sont sources d'interrogation : «Je n'ai jamais cessé de me demander ce qui m'est arrivé », ajoute-t-elle. Cette femme se retrouve abandonnée de/par tous et contrainte à partir sans aucun bien. Les conséquences qui découlent de ces accusations sont variées dans leur nombre et dans leur gravité et touchent les personnes accusées elles-mêmes et les membres de leurs familles :

- atteintes profondes et durables des victimes dans leur dignité, leur honneur, et leur réputation. «On ne peut pas parler de dignité quand on vous traite comme si vous n'étiez pas humain »;

- dissuasion des prétendants à venir prendre les enfants des victimes en mariage ou des filles d'autres familles à accepter de s'unir avec leurs garçons. «Ils ont même menti pour dire à l'ami de ma fille qu'elle était elle aussi sorcière et qu'il courait un danger s'il la prend pour épouse »;

- destruction des biens et habitations : "Quand ils ont encerclé ma maison, certains ont commencé à la détruire alors que je m'apprêtais à partir...»;

- violences, vindicte populaire, blessures graves, mort : « Nous étions un groupe de trois personnes que le charlatan a désignées comme étant des sorcières à la suite de deux décès dans la même journée et dans le même village. Les gens étaient en colère et nous violentaient. Une d'entre nous a reçu un gros caillou sur sa tête et est décédée sur place. Moi je me suis sauvée en prenant la direction de la brousse. Je ne me suis plus jamais retournée. Tout est resté et je ne veux plus jamais mettre pied dans ce village. » « Une femme du quartier m'a déshabillée à moitié avant qu'elle ne me bouscule et que je tombe. Après je ne sais plus combien de personnes m'ont donné des coups. Je ne sais pas comment j'ai pu survivre à tout cela (...) »;

- refus de la moindre assistance et du moindre geste de solidarité... « J'ai marché pendant toute la journée. J'avais très soif. Je suis allée demander de l'eau pour boire. Mais, un homme du village qui me suivait pour s'assurer que j'étais vraiment partie a dit de ne pas m'aider parce que je suis dangereuse. Les gens ont eu peur et m'ont dit de quitter leur village ».

\section{Discussion}

Cette recherche visait à étudier l'existence d'un lien entre les violences et la vulnérabilité chez la femme âgée en contexte burkinabé. Les résultats enregistrés confortent l'hypothèse formulée et montrent que les femmes exclues de leur communauté pour accusation de sorcellerie sont exposées à divers autres types de violences. La personne accusée est mise à l'index et regardée sans réserve comme coupable. Ces accusations, qui revêtent un 
caractère violent, sont les manifestations les plus visibles de la croyance à la sorcellerie. En dépit des instruments juridiques ratifiés par le Burkina Faso, les accusations de sorcellerie exposent les vieilles femmes à l'exclusion et à la déchéance sociales. Des traitements dégradants continuent d'être infligés à des femmes âgées, fragiles et sans défense qu'on accuse de sorcellerie ou de «mangeuses d'âmes ». Ces résultats confirment les constats antérieurs de la vivacité de la croyance en la sorcellerie et ses diverses conséquences psychologiques (Robert-Ouvray, 2008a ; Robert-Ouvray, 2008b ; Matlin, 2007 ; Trainar, 2006). Ils confirment les difficultés auxquelles sont confrontés les différents acteurs de la lutte contre ce phénomène, en raison de son caractère «insaisissable»: les affaires de sorcellerie échappent en bonne partie aux juridictions instituées par l'État et font l'objet d'un traitement plus clandestin par la justice villageoise (Fancello \& Bonhomme, 2018). En effet, relèvent ces auteurs, les institutions appréhendent la sorcellerie à partir de logiques qui leur sont propres : elles la jugent d'après les normes, les codes et les valeurs qui régissent leur fonctionnement et elles en parlent dans une langue et selon un vocabulaire toujours spécifiques (Fancello \& Bonhomme, 2018).

L'exclusion sociale pour fait de sorcellerie expose les femmes qui en sont victimes à la vulnérabilité, au regard du peu d'égard accordé à leurs droits à la vie, à la dignité, au respect, et à la sécurité. Or, ainsi que l'a relevé Hicks (2017), rien ne saurait satisfaire le besoin d'appartenance, le sentiment de réconfort et de sécurité que procure le fait d'être intégré. C'est le sens des analyses faites par Piazza (2008) et Bouisson (2011). Ces auteurs ont ressorti l'idée d'une angoisse du vieillissement que chacun(e) ressent. Lorsque dans le même temps on est coupé de son milieu socio-culturel d'appartenance pour accusation de sorcellerie, cela représente indéniablement une souffrance supplémentaire. Aucune possibilité n'est offerte aux victimes de tisser des liens sociaux qui font la toile de l'humanité et qui rendent envisageable de vivre pleinement. Elles perdent ainsi les moyens de rester en lien avec les supports et les divers véhicules de leur culture d'appartenance (Bouisson, 2011). Fragiles et incapables de se défendre (Ezembié, 2017), elles sont confrontées à un manque de moyens matériels et à une incapacité à s'intégrer à la société sur les plans social, économique, politique et culturel. Or, c'est un droit pour chacun(e) de nous d'attendre de sa culture qu'elle le soutienne et l'accompagne dans un processus qui commence dès le début de l'existence et qui ne cesse qu'avec la mort. Ce soutien se fera à travers des modèles, des scénarios divers, des politiques stimulantes (Bouisson, 2011).

L'exclusion sociale est une mise à mort psychologique et sociale qui peut conduire à la mort physique. «Durant la vieillesse, l'individu a particulièrement besoin de stabilité dans son entourage et aussi de sécurité 
dans ses relations émotionnelles pour maintenir son équilibre psychologique » (Giroux \& Payette, 1966, cités par Chagnon, 1990, p.496).

\section{Conclusion}

$\mathrm{Au}$ terme de cette recherche, il ressort que dans le contexte burkinabè, les croyances en la sorcellerie restent vivaces. Des accusations de sorcellerie sont régulièrement portées à l'encontre des femmes fragilisées par le poids de l'âge et qui sont, par ailleurs, sans défense ni soutien. Ces accusations sont suivies de violences de tous types (physique, verbale, psychologique) et de différentes orientations (directe vs indirecte). La rupture violente et brutale des liens socio-familiaux inscrit ces femmes au registre de la déchéance et de l'exclusion sociales et les confronte immédiatement à la vulnérabilité. L'intérêt de la présente recherche est d'interpeler sur la réalité des violences dont les femmes âgées sont victimes à la suite d'accusations de sorcellerie.

Cette recherche ne nourrit pas la prétention d'avoir épuisé le sujet. De futures recherches peuvent s'y intéresser pour davantage de résultats qui donneront les moyens de circonscrire et de contenir ce phénomène dans ses expressions et son extension. Pour le moment, on retient que la perte d'un être cher est une épreuve dont le poids se dédouble à l'idée de savoir que cette perte est le fait (réel ou supposé) d'autrui. La délicatesse du problème de la sorcellerie réside dans l'impossible production de preuves convaincantes pour accompagner ou étayer les accusations faites à certaines personnes pendant que les conséquences qui en découlent sont d'une gravité indéniable. L'on peine encore, au Burkina Faso, à formuler une réponse efficace et appropriée à la question des accusations de sorcellerie et de leurs conséquences. Il apparaît alors opportun et nécessaire d'assurer, d'une façon pérenne, une éducation citoyenne des populations et de les sensibiliser aux effets néfastes de l'exclusion sociale. Ce nécessaire recadrage inciterait les individus à prendre du recul par rapport à leurs croyances et à relativiser. Il s'agirait de travailler sur les mécanismes de représentations, les croyances, les systèmes de valeurs, de sorte à amener à une évolution des attitudes et des comportements dans le sens d'une plus grande connaissance/maîtrise de soi et $\mathrm{du}$ respect de l'autre. Cette éducation au changement des croyances devra prendre en compte la solidité, l'ancienneté, le caractère vital ou non de la croyance (Briefer \& Briefer, 2003).

\section{References:}

1. Airagnes, G. (2014). Existe-t-il des facteurs de vulnérabilité psychologique au fait d'être victime de violence? European $\begin{array}{lll}\text { Psychiatry, 29(8), 627-628. } & \text { DOI : }\end{array}$ https://doi.org/10.1016/j.eurpsy.2014.09.124. 
2. Amselle, J.L. (2014). Sorcellerie et sociétés secrètes, refuges contre la colonisation, Le Monde des Religions, Hors-Série, $\mathrm{N}^{\circ} 22$, 50-53.

3. Boa, T. R. (2010). La sorcellerien'existe pas, Les Editions du CERAP.

4. Bouisson, J. (2011). Vieillesse et vulnérabilité, dans J. Bouisson, C. Brisset, I. Tournier \& C. Vion (dir), Vieillissement \& vieillesse, vulnérabilité \& ressources : regards croisés. Maison des Sciences de l'Homme d'Aquitaine, (p.67-77).

5. Briefer, F. \& Briefer, J.P. (2003). Santé : croyances, théories et dogmes, ARS Medici, 25-26, 1-4.

6. Brodiez-Dolino, A. (2013). Vulnérabilités sanitaires et sociales, Le Mouvement Social, 1 (242), 3-8.

7. Chagnon, M. (1990). Psychologie. Le développement de la personne, Guérin.

8. Clery-Melin, P. (2014). Vulnérabilité psychique de l'avancée en âge, European Psychiatry, 29 (3), 575-576, DOI: https://doi.org/10.1016/j.eurpsy.2014.09.269.

9. Coakley, L. (2015). Impact de la sorcellerie en Afrique francophone subsaharienne : des femmes agissantes dans les nouvelles de Florent Couao-Zotti et d'Éveline Mankou. Master Arts et Etudes Francophones. Université Waterloo.

10. Crampton, A. (2013). No Peace in the House : Witchcraft Accusations as an "Old Woman's Problem" in Ghana. Anthropology \& Aging Quarterly, 34 (2), 199-2012.

11. Erny, P., La sorcellerie et nous, Conférence prononcée dans le cadre de l'Université Populaire de Colmar le 15 mars 1979 http://www.revue-des-sciences-sociales.com/pdf/rss08-erny2.pdf.

12. Ezembié, F. (2017). L'enfant africain et son univers, Karthala.

13. Fancello, S. (2015). Penser la sorcellerie en Afrique : un défi pour les sciences sociales ? Dans S. Fancello (dir.), Penser la sorcellerie en Afrique, (p. 21-43), Hermann.

14. Fancello, S. \& Bonhomme, J. (2018). L'État et les institutions face à la sorcellerie, Cahiers d'Études africaines, 3-4 (231-232), 573-591.

15. Fasse, L., Flahault, C., Bredart, A., Dolbeault, S. \& Sultan, S. (2012). Deuil et vulnérabilité psychique chez les personnes âgées, PsychoOncologie, 6, 231-244.

16. Flynn, C., Damant, D. \& Bernard, J. (2014). Analyser la violence structurelle faite aux femmes à partir d'une perspective féministe intersectionnelle, Nouvelles pratiques sociales, 26(2), 28-43. DOI :10.7202/1029260ar.

17. Hicks, D. (2017). Eloge de la dignité, Nouveaux Horizons. 
18. Kibuga, K. F. \& Dianga, A. (2000). Victimisation and killing of older women: witchcraft in Magu district, Tanzania. Southern African Journal of Gerontology, 9(2), 29-32.

19. Martinelli, B. \& Bouju, J. (dir.) (2012). Sorcellerie et violence en Afrique, Karthala.

20. Matlin, M. (2007). Psychologie des femmes, De Boeck.

21. Mbanzoulou, P. (2004). La violence à l'école : un retour d'expérience, Journal du droit des jeunes, 1 (231), 13-18.

22. Owusu, E. S. (2020). The Superstition that Maims the Vulnerable : Establishing the Magnitude of Witchcraft-Driven Mistreatment of Children and Older Women in Ghana. International Annals of Criminology, 58, 253-290.

23. Piazza, S. D. (2008). Handicap congénital et vieillissement, De Boeck.

24. Richard, J. (2004). Résilience et vulnérabilité : De l'ajustement des concepts en psychogérontologie, Gérontologie et société, 27 (109), 109-125.

25. Robert-Ouvray, S. (2008a). Les violences physiques, dans F. Caillard et M. Cocude (dir), Psychologie de l'éducation, Tome 2 : Famille et milieux de vie, (p.176-179), Bréal.

26. Robert-Ouvray, S. (2008b). Les violences psychologiques, dans F. Caillard et M. Cocude (dir), Psychologie de l'éducation, Tome 2: Famille et milieux de vie, (p.188-190), Bréal.

27. Roxburgh, S. (2016). Witchcraft and Violence in Ghana. An Assessment of Contemporary Mediation Efforts. Cahiers d'Études africaines, 4 (224), 891-914.

28. Sorel, O. et Dieu, E. (2013). Travailler sur la violence, ou la nécessité d'adopter un regard théorique, dans O. Sorel et E. Dieu (dir), Psychologie de la violence, (p.154-173), Studyrama.

29. Trainar, P. (2006). L'exclusion sociale, Commentaire, 3(115), 707716.

30. Van Caeneghem, R. (1955). Hekserij bij de Baluba van Kasai. Bruxelles : Académie Royale des Sciences Coloniales. 